\title{
Laplacian of the distance function on the cut locus on a riemannian manifold
}

\author{
François Générau
}

December 2, 2019

\begin{abstract}
In this note, we prove that, on a riemannian manifold $M$, the laplacian of the distance function to a point $b$ is $-\infty$ in the sense of barriers, at every point of the cut locus of $M$ with respect to $b$. We apply this result to an obstacle-type variational problem with the distance function as an obstacle, in order to replace it with a smoother obstacle.
\end{abstract}

Keywords: distance function, cut locus, obstacle problem, Riemannian manifold.

\section{Introduction}

Let $M$ be a Riemannian manifold of dimension $n \geq 2$ without boundary, and $b$ a point in $M$. We denote by $d$ the distance function on $M$ and $d_{b}$ the distance function to the point $b$. Let $T>0$ and $\gamma:[0, T] \rightarrow M$ be a unit speed geodesic such that $\gamma(0)=b, t_{0} \in(0, T)$ and $p=\gamma\left(t_{0}\right)$. We say that $p$ is a cut point of $b$ along $\gamma$ if $\gamma$ is length minimizing between $b$ and $p$, but not after $p$, i.e $d_{b}(\gamma(t))=t$ for $t \leq t_{0}$, and $d_{b}(\gamma(t))<t$ for $t>t_{0}$. The cut locus of $M$ with respect to $b$, denoted by $C_{u t}(M)$, is defined as the set of all cut points of $b$. Some well known facts related to the cut locus include:

- $\operatorname{Cut}_{b}(M)$ is the set of points $p$ in $M$, such that either there exist at least two minimizing geodesics from $b$ to $p$, or the points $b$ and $p$ are conjugate (see for instance [9, proposition 4.1]),

- $\operatorname{Cut}_{b}(M)$ is the closure of the set of points $p$ in $M$, such that there exist at least two minimizing geodesics from $b$ to $p$,

- the function $d_{b}$ is smooth outside $C u t_{b}(m)$ (see [9, proposition 4.8]),

- given $p \in M$, if there are at least two minimizing geodesics from $b$ to $p$, then the function $d_{b}$ is not differentiable at $p$ (see [9, proposition 4.8]).

Our main result is the following theorem. It describes the non-smooth behavior of the distance function at each point of the cut locus, in terms of its laplacian, in the sense of barriers. 
Theorem 1.1. Let $M$ be a smooth manifold without boundary of dimension $n \geq 2$, and $b \in M$. Let $d_{b}$ be the distance function to the point $b$, and $C u t_{b}(M)$ the cut locus with respect to $b$. The laplacian of $d_{b}$ on $C u t_{b}(M)$ is $-\infty$ in the sense of barriers. More precisely, for any $p \in C_{u} t_{b}(M)$ and $A>0$, there exists a smooth function $\phi$ defined on a neighborhood of $p$ such that

$$
\phi \geq d_{b}, \quad \phi(p)=d_{b}(p) \quad \text { and } \quad \Delta \phi(p) \leq-A .
$$

Remark 1.2. One could also prove an analog result for the cut locus with respect to a submanifold of $M$ instead of a point $b$. We restricted ourselves to this case for simplicity.

This result proves useful when one wants to apply the maximum principle to a function involving the distance function $d_{b}$. As an application, we use it to prove the corollary 1.3 below.

Our motivation is the following obstacle-type variational problem, and its relation with $\mathrm{Cut}_{b}(M)$ :

$$
\min _{\substack{u \in H^{1}(M) \\ u \leq d_{b}}} \int_{M}|\nabla u|^{2}-m \int_{M} u,
$$

where $m \in(0,+\infty)$. This problem can be seen as an extension to manifolds of the elastic-plastic torsion problem, which was extensively studied in the 60's and 70's - see for instance [1] (Cafarelli and Friedman), and the references therein. Indeed, the original elastic-plastic torsion problem boils down to the following problem:

$$
\min _{\substack{u \in H_{0}^{1}(\Omega) \\ u \leq d_{\partial \Omega}}} \int_{\Omega}|\nabla u|^{2}-m \int_{\Omega} u,
$$

where $\Omega$ is a bounded lipschitz open subset of $\mathbb{R}^{n}$, and $d_{\partial \Omega}$ the distance function to its boundary. The analog of the cut locus in this flat setting is called the ridge of $\Omega$ in [1. It is the closure of the set of points of $\Omega$ that have at least two closest points on the boundary $\partial \Omega$. Let $u_{m}$ and $v_{m}$ be the minimizers of (1.1) and $(1.2)$ respectively. In [1], using the fact that $\left|\nabla v_{m}\right| \leq 1$, the authors show that the ridge of $\Omega$ is contained in the set $\left\{v_{m}<d_{\partial \Omega}\right\}$. In problem (1.1), the inequality $\left|\nabla u_{m}\right| \leq 1$ doesn't hold in general. However, using the theorem 1.1 above, we can still prove that

$$
C u t_{b}(M) \subset\left\{u_{m}<d_{b}\right\} .
$$

Indeed, theorem 1.1 yields the following:

Corollary 1.3. For any $m>0$, there exists a function $\widetilde{d}_{b}$ that is smooth on $M \backslash\{b\}$, such that $u_{m} \leq \widetilde{d}_{b} \leq d_{b}, \widetilde{d}_{b}=d_{b}$ in a neighborhood of $b$ and $\widetilde{d}_{b}<d_{b}$ on $C_{u t}(M)$. In particular, the solution $u_{m}$ of $(1.1)$ is also the solution of the following variational problem with a smooth obstacle:

$$
\min _{\substack{u \in H^{1}(M) \\ u \leq \widetilde{d}_{b}}} \int_{M}|\nabla u|^{2}-m \int_{M} u .
$$


In turn, this corollary implies 1.3 . What is more, it can be used to show that, with the regularity theory for obstacle-type problems, $u_{m}$ is locally $C^{1,1}$ regular. For a broader study of problem (1.1) and its relation with the cut locus, we refer to 4 .

Note that in [7] (and in particular theorem 2.10), C.Mantegazza, G.Mascellani and G.Uraltsev gave a complete description of the distributional hessian and laplacian of $d_{b}$ using geometric measure theory tools. Here we are interested in inequalities in the sense of barriers, and we use mainly tools from riemannian geometry.

This paper is organized as follows. In section 2, we give a proof of theorem 1.1. In section 3 , we apply the theorem to prove corollary 1.3 .

Acknowledgement. We would like to thank Bozhidar Velichkov for some very useful discussions and ideas used in this paper.

\section{Proof of theorem 1.1}

Proof. Let $p \in \operatorname{Cut}_{b}(M)$ and $A>0$. We know that either there exist two minimizing geodesics from $b$ to $p$, or there exists a unique minimizing geodesic from $b$ to $p$ along which the two points are conjugate.

Case one. There exist two minimizing geodesics from $b$ to $p$. This implies that $d_{b}$ is not differentiable at $p$. Let $\delta>0$ be smaller than the distance between $p$ and $C u t_{p}(M)$, and $\psi: B(p, \delta) \rightarrow \mathbb{R}^{n}$ be a normal coordinate map around $p$. According to [8, Proposition 3.4], there exists $C>0$ such that the function $x \mapsto C|x|^{2}-d_{b} \circ \psi^{-1}(x)$ is convex. As it is not differentiable at 0 , it has at least two subgradients $v$ and $w$. We have

$$
C|x|^{2}-d_{b} \circ \psi^{-1}(x) \geq \max (v \cdot x, w \cdot x)-d_{b}(p),
$$

with equality for $x=0$. For $B>0$ to be chosen big enough later, let us define the function $f: \mathbb{R}^{n} \rightarrow \mathbb{R}$ by

$$
f(x):=\frac{1}{2}(v \cdot x+w \cdot x)+B(v \cdot x-w \cdot x)^{2}-d_{b}(p) .
$$

Then for $x$ in a neighborhood of 0 we have

$$
\begin{aligned}
f(x) & =\max (v \cdot x, w \cdot x)-\frac{1}{2}|v \cdot x-w \cdot x|+B(v \cdot x-w \cdot x)^{2}-d_{b}(p) \\
& \leq \max (v \cdot x, w \cdot x)-d_{b}(p)
\end{aligned}
$$

with equality at $x=0$. Setting $\phi=C|\psi|^{2}-f \circ \psi$, we get from (2.1) and (2.2) that for $q$ in a neighborhood of $p$,

$$
d_{b}(q) \leq \phi(q)
$$

with equality at $q=p$. What is more, as $\psi$ is a normal coordinate map, we have

$$
\Delta \phi(p)=\Delta\left(\phi \circ \psi^{-1}\right)(0)=2 n C-2 B|v-w|^{2} .
$$




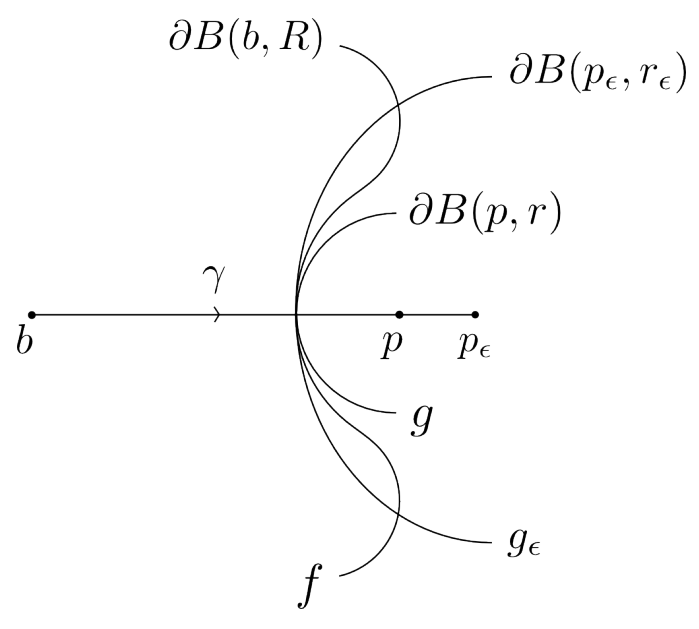

Figure 1: Construction of $r, R, q, p_{\epsilon}, r_{\epsilon}, f, g, g_{\epsilon}$.

In particular if $B$ is big enough, then we have $\Delta \phi(p) \leq-A$, which concludes case one.

Case two. There exists a unique minimizing geodesic $\gamma$ such that $\gamma(0)=b$ and $\gamma(1)=p$. For $r>0$ to be chosen small enough later, let $q$ be the intersection point of $\gamma$ with the sphere $\partial B(p, r)$, and $R=d_{b}(q)$. For $\epsilon>0$, let us define

$$
\begin{aligned}
p_{\epsilon} & :=\gamma(1+\epsilon), \\
r_{\epsilon} & :=r+d\left(p, p_{\epsilon}\right) .
\end{aligned}
$$

We may choose $r$ and $\epsilon$ so that $p$ and $p_{\epsilon}$ are contained in a totally normal geodesic ball $B(q, \delta)$ for some $\delta>0$. This implies in particular that for any points $p^{\prime}$ and $q^{\prime}$ in neighborhoods of $p$ and $q$ respectively, there exists a unique minimizing geodesic $\mu_{\left(p^{\prime}, q^{\prime}\right)}$ between $p^{\prime}$ and $q^{\prime}$, and $\mu_{\left(p^{\prime}, q^{\prime}\right)}$ depends continuously on $\left(p^{\prime}, q^{\prime}\right)$ (see [2, Remark 3.8]). Let us fix some normal coordinates at $q$ on $B(q, \delta)$ such that the unit normal vector to $\partial B(b, R)$ at $q$ is $\partial_{n}$. We will use these normal coordinates throughout the rest of the proof. In these coordinates, around $q, \partial B(b, R)$ is the graph of a smooth function $f$ such that $f(0)=0$ and $\nabla f(0)=0$. Likewise, $\partial B(p, r)$ and $\partial B\left(p_{\epsilon}, r_{\epsilon}\right)$ can be seen around $q$ as the graphs of some smooth functions $g$ and $g_{\epsilon}$ respectively, such that $g(0)=g_{\epsilon}(0)=0$ and $\nabla g(0)=\nabla g_{\epsilon}(0)=0$.

Step one. We prove that

$$
\exists v \in \mathbb{R}^{n-1}, v \neq 0, \quad v \cdot H f(0) v=v \cdot H g(0) v,
$$

where $H F$ denotes the hessian matrix of the function $F$. As the points $b$ and $p$ are conjugate, there exists a Jacobi field $J$ along $\gamma$ such that $J(0)=0, J(1)=0$ and $J(t) \neq 0$ for $t \in(0,1)$. We may extend it to a global smooth field $\widetilde{J}$ on $M$. 
Let $\left(\Phi_{\widetilde{J}}^{s}\right)_{s \in \mathbb{R}}$ be the flow of this vector field, defined by the following equation:

$$
\forall x \in M, \forall s \in \mathbb{R}, \frac{\mathrm{d}}{\mathrm{d} s} \Phi_{\widetilde{J}}^{s}(x)=\widetilde{J}\left(\Phi_{\widetilde{J}}^{s}(x)\right) .
$$

We define a variation $\Gamma$ of the curve $\gamma$ as follows:

$$
\forall s \in \mathbb{R}, \forall t \in[0,1], \Gamma(s, t):=\Phi_{\widetilde{J}}^{s}(\gamma(t)) .
$$

For any $s \in \mathbb{R}, \Gamma(s, \cdot)$ is still a curve from $b$ to $p$, as $\widetilde{J}(0)=0$ and $\widetilde{J}(1)=0$. As $\gamma$ is a geodesic, we have

$$
\frac{\mathrm{d}}{\mathrm{d} s}[\operatorname{length}(\Gamma(s, \cdot))]_{s=0}=0 .
$$

As $\Gamma$ is a fixed point variation of $\gamma$ whose variation field $J$ is a Jacobi field, we also have (see [6, Proposition 10.14] for instance).

$$
\frac{\mathrm{d}^{2}}{\mathrm{~d} s^{2}}[\operatorname{length}(\Gamma(s, \cdot))]_{s=0}=0 .
$$

By the implicit function theorem, for any $s$ close enough to 0 , there exist some unique times $t_{R}(s)$ and $t_{r}(s)$ such that

$$
d\left(\Gamma\left(s, t_{R}(s)\right), b\right)=R \quad \text { and } \quad d\left(\Gamma\left(s, t_{r}(s)\right), p\right)=r .
$$

Moreover, the functions $s \mapsto t_{R}(s)$ and $s \mapsto t_{r}(s)$ are smooth. Now we set

$$
M(s):=\Gamma\left(s, t_{R}(s)\right) \quad \text { and } \quad N(s):=\Gamma\left(s, t_{r}(s)\right) .
$$

We have

$$
\begin{aligned}
\operatorname{length}(\Gamma(s, \cdot))= & \operatorname{length}\left(\Gamma(s, \cdot)_{\left.\right|_{\left[0, t_{R}(s)\right]}}\right)+\operatorname{length}\left(\Gamma(s, \cdot)_{\left.\right|_{\left[t_{R}(s), t_{r}(s)\right]}}\right) \\
& \quad+\operatorname{length}\left(\Gamma(s, \cdot)_{\left[t_{r}(s), 1\right]}\right) \\
\geq d\left(\Gamma(s, 0), \Gamma\left(s, t_{R}(s)\right)\right)+d\left(\Gamma\left(s, t_{R}(s)\right), \Gamma\left(s, t_{r}(s)\right)\right) & \quad+d\left(\Gamma\left(s, t_{r}(s)\right), \Gamma(s, 1)\right) \\
= & +d(M(s), N(s))+r .
\end{aligned}
$$

Meanwhile, as $s$ goes to 0 , thanks to 2.4, we also have

$$
\operatorname{length}(\Gamma(s, \cdot))=\operatorname{length}(\gamma)+o\left(s^{2}\right)=R+r+o\left(s^{2}\right),
$$

so $d(M(s), N(s)) \leq o\left(s^{2}\right)$. In our normal coordinates around $q$, we have $d(x, y) \geq$ $c|x-y|$ where $c>0$ is a constant and $|\cdot|$ is the euclidean norm. This comes from the fact that the metric $g$ of $M$ and the euclidean metric are locally equivalent in any coordinates system. With this remark, we get

$$
M(s)=N(s)+o\left(s^{2}\right),
$$


where the equality is to be understood in coordinates. For any $s$ small, let $x_{M}(s), x_{N}(s) \in \mathbb{R}^{n-1}$ be such that $M(s)=\left(x_{M}(s), f\left(x_{M}(s)\right)\right)$ and $N(s)=$ $\left(x_{N}(s), g\left(x_{N}(s)\right)\right)$. The functions $x_{M}$ and $x_{N}$ are smooth because $M$ and $N$ are. Using the fact that $\nabla f(0)=\nabla g(0)=0,2.5$ gives

$$
x_{M}^{\prime}(0) \cdot H f(0) x_{M}^{\prime}(0)=x_{N}^{\prime}(0) \cdot H g(0) x_{N}^{\prime}(0) .
$$

Let us show that $x_{N}^{\prime}(0)=x_{M}^{\prime}(0)$. We have

$$
\begin{aligned}
M^{\prime}(0) & =\partial_{s} \Gamma\left(0, t_{R}(0)\right)+\partial_{t} \Gamma\left(0, t_{R}(0)\right) t_{R}^{\prime}(0) \\
& =J\left(t_{R}(0)\right)+\dot{\gamma}\left(t_{R}(0)\right) t_{R}^{\prime}(0) .
\end{aligned}
$$

By definition the curve $M$ is on the sphere $\partial B(b, R)$, so $M^{\prime}(0)$ is tangent to this sphere and orthogonal to $\dot{\gamma}\left(t_{R}(0)\right)$ by the Gauss lemma. Also, as $J(0)=0$ and $J(1)=0$, the Jacobi field $J$ is normal to $\gamma$ (see [6, lemma 10.6]), so $J\left(t_{R}(0)\right.$ ) is orthogonal to $\dot{\gamma}\left(t_{R}(0)\right)$. Combined with these facts, 2.7) yields $M^{\prime}(0)=$ $J\left(t_{R}(0)\right)$. Likewise, we have $N^{\prime}(0)=J\left(t_{r}(0)\right)=J\left(t_{R}(0)\right)$. So $M^{\prime}(0)=N^{\prime}(0)$, and consequently $x_{M}^{\prime}(0)=x_{N}^{\prime}(0)$. What is more, as $J(t) \neq 0$ for $t \in(0,1)$, we have $x_{M}^{\prime}(0) \neq 0$. So setting $v=x_{M}^{\prime}(0)$, with (2.6) we get (2.3).

Step two. Now we want to show that

$$
v \cdot H g(0) v>v \cdot H g_{\epsilon}(0) v .
$$

Let us first show that $g \geq g_{\epsilon}$ in a neighborhood of 0 . Let us argue by contradiction and assume that for some $x \in \mathbb{R}^{n-1}$, we have $g(x)<g_{\epsilon}(x)$. Let $\mu:[0,1] \rightarrow$ $M$ be the shortest geodesic from the point of coordinate $(x, g(x))$ to the point of coordinate $(0, r)$, i.e. $p$. If $x$ has been taken close enough to 0 , then for $t \in[0,1]$, $\mu(t)$ stays inside the normal neighborhood of $q, B(q, \delta)$, on which our normal coordinates are defined. For $t \in[0,1]$, let $x(t) \in \mathbb{R}^{n-1}$ and $z(t) \in \mathbb{R}$ be such that in coordinates, $\mu(t)=(x(t), z(t))$. We have $z(0)=g(x)<g_{\epsilon}(x)=g_{\epsilon}(x(0))$. And $z(1)=r>0=g_{\epsilon}(0)=g_{\epsilon}(x(1))$. As $\mu$ is continuous, there exists $t \in(0,1)$ such that $z(t)=g_{\epsilon}(x(t))$, i.e. $\mu(t) \in \partial B\left(p_{\epsilon}, r_{\epsilon}\right)$. This implies

$$
\begin{aligned}
r_{\epsilon} & =d\left(\mu(t), p_{\epsilon}\right) \\
& \leq d(\mu(t), p)+d\left(p, p_{\epsilon}\right) \\
& <d(\mu(0), p)+d\left(p, p_{\epsilon}\right) \\
& =r+d\left(p, p_{\epsilon}\right) \\
& =r_{\epsilon},
\end{aligned}
$$

which gives a contradiction. We conclude that $g \geq g_{\epsilon}$. In particular, for any $w \in \mathbb{R}^{n-1}$, we have $w \cdot H g(0) w \geq w \cdot H g_{\epsilon}(0) w$. Thus, the matrix $H g(0)-H g_{\epsilon}(0)$ is symetric non-negative. To show that it is positive definite and conclude that 2.8 holds, we only need to show that

$$
\forall w \in \mathbb{R}^{n-1}, \quad H g(0) w \neq H g_{\epsilon}(0) w .
$$

To prove this, let us define the exponential map $\exp _{S}$ associated with a smooth oriented hypersurface $S$ of $M$. Let Exp be the global exponential map, defined 
on the whole tangent space of $M$. Let $\nu_{S}$ be the unit normal vector to $S$. We set

$$
\begin{aligned}
& \exp _{S}: S \times[0, \infty] \rightarrow \quad M \\
& (\theta, t) \quad \mapsto \operatorname{Exp}\left(t \nu_{S}(\theta)\right)
\end{aligned}
$$

The geodesic $\gamma$ is minimizing between $p_{\epsilon}$ and $\partial B\left(p_{\epsilon}, r_{\epsilon}\right)$. In particular, the point $p$ is not a cut point of $q$ along $\gamma$, so lemma 2.1 below shows that the differential of the map $\exp _{\partial B\left(p_{\epsilon}, r_{\epsilon}\right)}$ is invertible at $(q, r)$. On the contrary, the map $\exp _{\partial B(p, r)}(\cdot, r)$ is constant and so its differential is null at $q$. In particular, for any $w \in T_{q} \partial B(p, r)$, we have

$$
\nabla_{w} \nu_{\partial B\left(p_{\epsilon}, r_{\epsilon}\right)}(q) \neq \nabla_{w} \nu_{\partial B(p, r)}(q),
$$

where $\nabla_{w}$ denotes the covariant derivative in the direction $w$. Let us define the families of vectors $\left(u_{i}^{g}\right)_{1 \leq i \leq n-1}$ and $\left(u_{i}^{g_{\epsilon}}\right)_{1 \leq i \leq n-1}$ at the point of coordinates $(x, z) \in \mathbb{R}^{n-1} \times \mathbb{R}$, by $u_{i}^{g}(x, z):=\partial_{i}+\partial_{i} g(x) \partial_{n}$ and $u_{i}^{g_{\epsilon}}(x, z):=\partial_{i}+\partial_{i} g_{\epsilon}(x) \partial_{n}$. These vectors form a basis of the tangent spaces of $\partial B(p, r)$ and $\partial B\left(p_{\epsilon}, r_{\epsilon}\right)$ respectively, as we recall that these surfaces are the graphs of the functions $g$ and $g_{\epsilon}$. Furthermore, the two basis are identical at $(0,0)$. Thus, 2.10 is equivalent to

$$
\exists i \in\{1, . ., n-1\}, u_{i}^{g_{\epsilon}} \cdot \nabla_{w} \nu_{\partial B\left(p_{\epsilon}, r_{\epsilon}\right)}(q) \neq u_{i}^{g} \cdot \nabla_{w} \nu_{\partial B(p, r)}(q) .
$$

Note that if some vector fields $X$ and $Y$ are orthogonal, then we have $X \cdot Y=0$, and so $\nabla_{w} X \cdot Y+X \cdot \nabla_{w} Y=0$. So we get

$$
\begin{gathered}
\exists i \in\{1, . ., n-1\}, \nabla_{w} u_{i}^{g_{\epsilon}} \cdot \nu_{\partial B\left(p_{\epsilon}, r_{\epsilon}\right)}(q) \neq \nabla_{w} u_{i}^{g} \cdot \nu_{\partial B(p, r)}(q), \\
\text { i.e. } \quad \exists i \in\{1, . ., n-1\}, \nabla_{w} u_{i}^{g_{\epsilon}} \cdot \partial_{n} \neq \nabla_{w} u_{i}^{g} \cdot \partial_{n} .
\end{gathered}
$$

Recalling that in normal coordinates, the tangent vectors $\left(\partial_{j}\right)_{1 \leq j \leq n}$ have vanishing covariant derivatives at 0 , we get

$$
\exists i \in\{1, . ., n-1\}, w^{j} \partial_{j i} g_{\epsilon}(0) \neq w^{j} \partial_{j i} g(0),
$$

which is 2.9$)$. So $(2.8)$ holds.

Step three. Putting steps one and two together, we get

$$
\exists v \neq 0, \quad v \cdot\left(H g_{\epsilon}(0)-H f(0)\right) v<0 .
$$

In particular, there exists a basis $\left(v_{i}\right)_{1 \leq i \leq n-1}$ of $\mathbb{R}^{n-1}$ with $v_{1}=v$, that is orthogonal for the quadratic form $q_{\epsilon}(w)=w \cdot\left(H g_{\epsilon}(0)-H f(0)\right) w$. Let $k_{\epsilon}$ be a quadratic form of $\mathbb{R}^{n-1}$ such that the $\left(v_{i}\right)$ are orthogonal for $k_{\epsilon}, k_{\epsilon}(v)=0$ and for $i \geq 2, k_{\epsilon}\left(v_{i}\right)=\max \left(0, q_{\epsilon}\left(v_{i}\right)+1\right)$. This way we have $k_{\epsilon}>q_{\epsilon}$, and $k_{\epsilon} \geq 0$. Now let us set $h_{\epsilon}=g_{\epsilon}-k_{\epsilon}$. Note that in dimension 2 , we actually have $h_{\epsilon}=g_{\epsilon}$, and the rest of the proof is a bit less technical. By construction we have

$$
\begin{aligned}
& h_{\epsilon} \leq g_{\epsilon}, \\
& h_{\epsilon}=g_{\epsilon} \quad \text { on } \mathbb{R} v, \\
& h_{\epsilon} \leq f \quad \text { on } B\left(0, \rho_{\epsilon}\right), \text { for some } \rho_{\epsilon}>0,
\end{aligned}
$$


where 2.13) comes from the fact that the hessian of $h_{\epsilon}$ at 0 verifies $H h_{\epsilon}(0)=$ $H g_{\epsilon}(0)-k_{\epsilon}<H g_{\epsilon}(0)-q_{\epsilon}=H f(0)$, and $h_{\epsilon}(0)=f(0)=0, \nabla h_{\epsilon}(0)=\nabla f(0)=$ 0 . It will be convenient, at the end of the proof, to have a smooth function $h: \mathbb{R}^{n-1} \rightarrow \mathbb{R}$ such that

$$
h(0)=0, \quad \nabla h(0)=0, \quad \text { and for all } \epsilon>0 \text { sufficiently small, } \quad h_{\epsilon} \geq h .
$$

To see that such function exists, we note that for $\epsilon<\epsilon^{\prime}$, we have $g_{\epsilon} \geq g_{\epsilon^{\prime}}$. This can be proven the same way we proved $g_{\epsilon} \leq g$ at the beginning of step two. We then fix $\epsilon^{\prime}>0$. Therefore, for any $\epsilon$ sufficiently small, we have $g_{\epsilon^{\prime}} \leq g_{\epsilon} \leq g$. This allows us to see that the quadratic forms $q_{\epsilon}$, and then $k_{\epsilon}$, are bounded independently of $\epsilon$, by a quadratic form $k$. In turn, this implies that the function $h=g_{\epsilon^{\prime}}-k$ verifies (2.14). Let $S_{h_{\epsilon}}$ be the hypersurface of $M$ defined in our normal coordinates around $q$ as the graph of the function $h_{\epsilon}$ on $B\left(0, \rho_{\epsilon}\right)$. Let us set

$$
\phi_{\epsilon}=d\left(\cdot, S_{h_{\epsilon}}\right)+R .
$$

We will show that for $\epsilon$ sufficiently small, $\phi_{\epsilon}$ is the function $\phi$ we are looking for to prove the theorem.

Let us first show that $\phi_{\epsilon} \geq d_{b}$ in a neighborhood of $p$. Let $p^{\prime} \in B(q, \delta)$ be a point near $p$. Let $\mu:[0,1] \rightarrow M$ be a length minimizing geodesic between $S_{h_{\epsilon}}$ and $p^{\prime}$. For $t \in[0,1]$, let $(x(t), z(t)) \in \mathbb{R}^{n-1} \times \mathbb{R}$ be the coordinates of $\mu(t)$. As $\mu(0) \in S_{h_{\epsilon}}$, we have $z(0)=h_{\epsilon}(x(0)) \leq f(x(0))$. Moreover, the coordinates $(x, z)=(0, r)$ of $p$ verify $z>f(x)$. Therefore, provided $p^{\prime}$ is close enough to $p$, we have $z(1) \geq f(x(1))$. Thus, there exists $t \in[0,1]$ such that $z(t)=f(x(t))$, i.e. $\mu(t) \in \partial B(b, R)$. This implies

$$
d\left(\cdot, S_{h_{\epsilon}}\right) \geq d(\cdot, \partial B(b, R)) \quad \text { on a neighborhood of } p,
$$

and so $\phi_{\epsilon} \geq d_{b}$ on a neighborhood of $p$.

As $q \in S_{h_{\epsilon}}$, we also have $\phi_{\epsilon}(p) \leq d(p, q)+R=d_{b}(p)$. So we get $\phi_{\epsilon}(p)=d_{b}(p)$ as well.

We are left to show that $\phi_{\epsilon}$ is smooth around $p$, and that given $A>0$, we have $\Delta \phi_{\epsilon}(p) \leq-A$, provided $\epsilon$ has been taken small enough. Let us first show that $\phi_{\epsilon}$ is smooth around $p$. Between $p_{\epsilon}$ and $q$, the geodesic $\gamma$ is minimizing among all geodesics from $p_{\epsilon}$ to $\partial B\left(p_{\epsilon}, r_{\epsilon}\right)$. The same way we have shown that $h_{\epsilon} \leq f$ implies $d\left(\cdot, S_{h_{\epsilon}}\right) \geq d(\cdot, \partial B(b, R))$, one can show that $h_{\epsilon} \leq g_{\epsilon}$ implies

$$
d\left(\cdot, S_{h_{\epsilon}}\right) \geq d\left(\cdot, \partial B\left(p_{\epsilon}, r_{\epsilon}\right)\right) \quad \text { on a neighborhood of } p .
$$

Therefore, between $q$ and $p_{\epsilon}, \gamma$ is still minimizing among geodesics from $p_{\epsilon}$ to $S_{h_{\epsilon}}$. We may apply lemma 2.1 to conclude that $\phi_{\epsilon}$ is smooth at $p$. Now for $t$ in a neighborhood of 0 , let us define $c(t):=\exp _{\partial B\left(p_{\epsilon}, r_{\epsilon}\right)}\left(r, \exp _{q}\left(t v, g_{\epsilon}(t v)\right)\right)$. We have,

$$
\begin{aligned}
d\left(c(t), S_{h_{\epsilon}}\right) & \geq d\left(c(t), \partial B\left(p_{\epsilon}, r_{\epsilon}\right)\right) \quad \text { because of 2.16, } \\
& =d\left(c(t), \exp _{q}\left(t v, g_{\epsilon}(t v)\right)\right) \quad \text { because of lemma 2.1. } \\
& =d\left(c(t), \exp _{q}\left(t v, h_{\epsilon}(t v)\right)\right) \quad \text { because of 2.12, } \\
& \geq d\left(c(t), S_{h_{\epsilon}}\right) .
\end{aligned}
$$


Therefore, the first inequality is actually an equality:

$$
d\left(c(t), S_{h_{\epsilon}}\right)=d\left(c(t), \partial B\left(p_{\epsilon}, r_{\epsilon}\right)\right) .
$$

As $d\left(\cdot, S_{h_{\epsilon}}\right)$ and $d\left(\cdot, \partial B\left(p_{\epsilon}, r_{\epsilon}\right)\right)$ have the same gradient at $c(0)=p$, we deduce that

$$
\nabla_{\dot{c}(0), \dot{c}(0)}^{2}\left[d\left(\cdot, S_{h_{\epsilon}}\right)\right]_{p}=\nabla_{\dot{c}(0), \dot{c}(0)}^{2}\left[d\left(\cdot, \partial B\left(p_{\epsilon}, r_{\epsilon}\right)\right]_{p} .\right.
$$

We have $\dot{c}(0) \neq 0$ because of lemma 2.1 again. Setting $w_{1}:=\dot{c}(0) /|\dot{c}(0)|$, we get

$$
\nabla_{w_{1}, w_{1}}^{2}\left[d\left(\cdot, S_{h_{\epsilon}}\right)\right]_{p}=\nabla_{w_{1}, w_{1}}^{2}\left[d\left(\cdot, \partial B\left(p_{\epsilon}, r_{\epsilon}\right)\right]_{p} .\right.
$$

Let us complete $w_{1}$ into an orthonormal basis $\left(w_{1}, . ., w_{n}\right)$ of $T p M$. Let $S_{h}$ be the hypersurface of $M$ defined in our normal coordinates at $q$ as the graph of the function $h$ from (2.14). The same way we have proved (2.15), one can prove that $d\left(\cdot, S_{h_{\epsilon}}\right) \leq d\left(\cdot, S_{h}\right)$. As these two functions are equal up to order 1 at $p$, we deduce that

$$
\nabla_{w_{i}, w_{i}}^{2}\left[d\left(\cdot, S_{h_{\epsilon}}\right)\right]_{p} \leq \nabla_{w_{i}, w_{i}}^{2}\left[d\left(\cdot, S_{h}\right]_{p}\right.
$$

Moreover, we know from [8, proposition 3.4] that there exists a constant $C>0$ such that for all $i, \nabla_{w_{i}, w_{i}}^{2}\left[d\left(\cdot, S_{h}\right]_{p} \leq C\right.$, so we get

$$
\forall i \geq 2, \nabla_{w_{i}, w_{i}}^{2}\left[d\left(\cdot, S_{h_{\epsilon}}\right)\right]_{p} \leq C,
$$

where $C$ is independent of $\epsilon$. Combining this inequality with (2.17), we get

$$
\Delta \phi_{\epsilon}(p) \leq(n-1) C+\nabla_{w_{1}, w_{1}}^{2}\left[d\left(\cdot, \partial B\left(p_{\epsilon}, r_{\epsilon}\right)\right]_{p} .\right.
$$

Let us pick some normal coordinates $\left(x^{i}\right)_{1 \leq i \leq n}$ at $p_{\epsilon}$, such that we have $\partial_{n}=\dot{\gamma}$ on the curve $\gamma$. By the Gauss lemma, the set of points that are equidistant to $\partial B\left(p_{\epsilon}, r_{\epsilon}\right)$ are orthogonal to the geodesics starting out orthogonally from $\partial B\left(p_{\epsilon}, r_{\epsilon}\right)$. Therefore we have $\dot{c}(0) \cdot \dot{\gamma}(1)=0$, or equivalently $w_{1} \cdot \partial_{n}=0$. In particular, if $\left(w_{1}^{i}\right)$ are the coordinates of $w$ relatively to the normal coordinates $\left(x^{i}\right)$ at $p_{\epsilon}$, we have $w_{1}^{n}=0$. In these coordinates we have $d\left(x, \partial B\left(p_{\epsilon}, r_{\epsilon}\right)\right)=$ $r_{\epsilon}-|x|$, so

$$
\begin{aligned}
\nabla_{w_{1}, w_{1}}^{2}\left[d\left(\cdot, \partial B\left(p_{\epsilon}, r_{\epsilon}\right)\right](x)\right. & =-\nabla_{w_{1}, w_{1}}^{2}|x| \\
& =-w_{1}^{i} w_{1}^{j}\left(\partial_{i j}|x|-\Gamma_{i j}^{k} \partial_{k}|x|\right) \\
& =-w_{1}^{i} w_{1}^{j}\left(-\frac{\delta_{i j}}{|x|}+\frac{x_{i} x_{j}}{|x|^{3}}+\Gamma_{i j}^{k}(x) \frac{x_{k}}{|x|}\right),
\end{aligned}
$$

where the $\left(\Gamma_{i j}^{k}\right)$ are the Christoffel symbols. We apply this formula at the point $p$ of coordinates $\left(0, . ., 0,-d\left(p, p_{\epsilon}\right)\right)$, and obtain

$$
\nabla_{w_{1}, w_{1}}^{2}\left[d\left(\cdot, \partial B\left(p_{\epsilon}, r_{\epsilon}\right)\right](p)=\frac{w_{1}^{i} w_{1}^{j} \delta_{i j}}{d\left(p, p_{\epsilon}\right)}-w_{1}^{i} w_{1}^{j} \Gamma_{i j}^{n}(p) .\right.
$$


As we are in normal coordinates, there exist a continuous function $\rho:[0,+\infty) \rightarrow$ $[0,+\infty)$ such that $\rho(0)=0$ and

$$
\left|\Gamma_{i j}^{k}(x)\right| \leq \rho(|x|) \quad \text { and } \quad\left|g^{i j}(x)-\delta_{i j}\right| \leq|x| \rho(|x|) .
$$

Moreover, note that we only consider coordinates centered at some points $p_{\epsilon}$ contained in a bounded neighborhood of $p$, so the function $\rho$ is independent of $\epsilon$. With these remarks, 2.19 yields

$$
\mid \nabla_{w_{1}, w_{1}}^{2}\left[d\left(\cdot, \partial B\left(p_{\epsilon}, r_{\epsilon}\right)\right](p)-\frac{|w|^{2}}{d\left(p, p_{\epsilon}\right)} \mid \leq 2\left(\sum_{i} w_{1}^{i}\right)^{2} \rho\left(d\left(p, p_{\epsilon}\right)\right) .\right.
$$

As $\epsilon \rightarrow 0$, the right hand side goes to 0 , and $\frac{|w|^{2}}{d\left(p, p_{\epsilon}\right)}=\frac{1}{d\left(\left(p, p_{\epsilon}\right)\right)} \rightarrow+\infty$, so

$$
\nabla_{w_{1}, w_{1}}^{2}\left[d\left(\cdot, \partial B\left(p_{\epsilon}, r_{\epsilon}\right)\right](p) \rightarrow-\infty\right.
$$

which concludes the proof with 2.18).

The following lemma is a well known fact when the hypersurface $S$ considered is replaced by a point. We give a proof here, as we couldn't find it in the literature.

Lemma 2.1. Let $S$ be a smooth hypersurface of $M$ such that $\bar{S}$ is compact. Let $\mu:[0,1] \rightarrow M$ be a geodesic such that $q:=\mu(0) \in S$. We assume that $\mu$ is length minimizing between $p:=\mu(1)$ and $\bar{S}$, and that $p$ is not a cut point of $q$ along $\mu$. Then the exponential map $\exp _{S}$ is a diffeomorphism from a neighborhood $U(q)$ of $q$ to a neighborhood $U(p)$ of $p$, and on $U(p)$ we have

$$
d(\cdot, S)=\pi_{2} \circ\left(\exp _{S_{\mid U(q)}}\right)^{-1},
$$

where $\pi_{2}: S \times \mathbb{R} \rightarrow \mathbb{R}$ is the projection on the second coordinate. In particular, $d(\cdot, S)$ is smooth around $p$.

Proof. As $p$ is not a cut point of $q$ along $\gamma$, it is not a focal point of $S$ (see [9, lemma 2.11]). In particular, $\exp _{S}$ is a local diffeomorphism from $(q, r)$ to $p$. As $q$ is the unique closest point to $p$ on the compact set $\bar{S}$, for any neighborhood $U(q)$ of $q$, there exists a neighborhood $U(p)$ of $p$ such that for any point $p^{\prime} \in U(p)$ the closest points to $p^{\prime}$ on $\bar{S}$ are inside $U(q)$. We choose such $U(p)$ and $U(q)$ so that $\exp _{S}$ is a diffeomorphism from $U(q)$ to $U(p)$. Let $p^{\prime} \in U(p)$ and $r:=d\left(p^{\prime}, S\right)$. A closest point to $p^{\prime}$ on $S$ is a point $q^{\prime} \in U(q)$ such that $p^{\prime}=\exp _{S}\left(q^{\prime}, r\right)$, and so $d\left(p^{\prime}, S\right)=r=\pi_{2} \circ\left(\exp _{S_{\mid U(q)}}\right)^{-1}\left(p^{\prime}\right)$. This concludes the proof.

\section{Application to a variational problem}

Recall that $u_{m}$ is a minimizer in 1.1. We give a proof of corollary 1.3 . 
Proof of corollary 1.3. It is a consequence of the minimality of $u_{m}$ in 1.1 that we have in the sense of distributions:

$$
\Delta u_{m} \geq-m \text {. }
$$

This implies, from the theory of subharmonic distributions, that $u_{m}$ has an upper semi-continuous representative. It is proved in [5, section 9], in the present setting of manifolds. For a reference about subharmonic functions in the euclidean space, see for instance [3].

Let $x \in C u t_{b}(M)$. According to theorem 1.1 we can find a smooth function $\phi$ defined on an open neighborhood $N$ of $x$ such that:

$$
\phi \geq d_{b}, \quad \phi(x)=d_{b}(x) \text { and } \quad \Delta \phi \leq-m-1 .
$$

Then the function $u_{m}-\phi$ is non-positive strictly subharmonic on $N$ and so by the maximum principle for subharmonic functions, we have

$$
\left(u_{m}-\phi\right)(x)<0, \quad \text { i.e. } \quad u_{m}(x)<d_{b}(x) .
$$

Thus, the function $u_{m}-d_{b}$ is upper semi-continuous and negative on the compact set $C_{u t}(M)$, so there exists $\epsilon>0$ such that the set $\left\{u_{m}<d_{b}-\epsilon\right\}$ is an open set containing $C u t_{b}(M)$. Now choose any smooth bump function $\rho: M \rightarrow[0,1]$ that is compactly supported in $\left\{u_{m}<d_{b}-\epsilon\right\} \backslash\{b\}$ and such that $\rho=1$ on $\mathrm{Cut}_{b}(M)$, and a smooth function $d_{b, \epsilon}: M \rightarrow \mathbb{R}$ such that

$$
\left\|d_{b, \epsilon}-\left(d_{b}-\frac{\epsilon}{2}\right)\right\|_{L^{\infty}}<\frac{\epsilon}{2} .
$$

We set

$$
\widetilde{d}_{b}:=(1-\rho) d_{b}+\rho d_{b, \epsilon} .
$$

It is now an easy exercise to show that $\widetilde{d_{b}}$ verifies the desired conditions.

\section{References}

[1] Luis A Caffarelli and Avner Friedman. The free boundary for elasticplastic torsion problems. Transactions of the American Mathematical Society, 252:65-97, 1979.

[2] Manfredo Perdigao do Carmo. Riemannian geometry. Birkhäuser, 1992.

[3] Jean-Pierre Demailly. Complex analytic and differential geometry, 2007.

[4] François Générau, Edouard Oudet, and Bozhidar Velichkov. Approximation of the cut locus with a variational problem. In preparation.

[5] F Reese Harvey and H Blaine Lawson. The equivalence of viscosity and distributional subsolutions for convex subequations - a strong bellman principle. Bulletin of the Brazilian Mathematical Society, New Series, 44(4):621$652,2013$. 
[6] John M Lee. Riemannian manifolds: an introduction to curvature, volume 176. Springer Science \& Business Media, 2006.

[7] Carlo Mantegazza, Giovanni Mascellani, and Gennady Uraltsev. On the distributional hessian of the distance function. Pacific Journal of Mathematics, 270(1):151-166, 2014.

[8] Carlo Mantegazza and Andrea Carlo Mennucci. Hamilton-jacobi equations and distance functions on riemannian manifolds. Applied Mathematics $\&$ Optimization, 47(1), 2003.

[9] Takashi Sakai. Riemannian geometry, volume 149. American Mathematical Soc., 1996.

François Générau: Laboratoire Jean Kuntzmann (LJK), Université Joseph Fourier Bâtiment IMAG, 700 avenue centrale, 38041 Grenoble Cedex 9 - FRANCE

francois.generau@univ-grenoble-alpes.fr 